\title{
Studies about the Atomic Capabilities Concept for Linear Control Systems in Physical Multi-Agent Environments
}

\author{
Christian G. Quintero M., Josep Ll. de la Rosa, Josep Vehí \\ Department of Electronics, Computer Science and Automatic Control \\ University of Girona, Catalonia, Spain \\ \{cgquinte,peplluis,vehi\}@eia.udg.es
}

\begin{abstract}
This paper shows the impact of the atomic capabilities concept to include control-oriented knowledge of linear control systems in the decisions making structure of physical agents. These agents operate in a real environment managing physical objects (e.g. their physical bodies) in coordinated tasks. This approach is presented using an introspective reasoning approach and control theory based on the specific tasks of passing a ball and executing the offside manoeuvre between physical agents in the robotic soccer testbed. Experimental results and conclusions are presented, emphasising the advantages of our approach that improve the multi-agent performance in cooperative systems.
\end{abstract}

Index Terms - Intelligent systems, Co-operative robotics, Mobile robotics, Agent technology, Neural networks

\section{INTRODUCTION}

Physical agents (e.g. mobile robots) that operate in a real environment managing physical objects (e.g. their physical bodies) lack accurate knowledge about the physical features of their bodies. This lack results in a loss of performance in cooperative decisions in coordinated tasks. This lack of information is caused by the absence of appropriate representations of the physical features of physical objects (e.g. their dynamics), namely, the absence of an agents-oriented representation of the specifications of automatic controllers.

The atomic capabilities concept is our proposal aimed at closing the gap between the agents and the low abstraction level of automatic control architectures. The idea is that the atomic capabilities include control-oriented knowledge about the specifications, structure and other relevant details that are encapsulated in every controller. Otherwise, this specific knowledge about every controller is not taken into account and never reused in order to achieve better agent cooperation. This cooperation is measured in terms of physically grounded and reliable commitments which result in a group of cooperating agents performing better.

In this research line, some authors have studied the problems related to the control, coordination and cooperation between physical agents (agents + physical bodies) when executing coordinated tasks, taking into account the dynamics of their physical bodies, but a general formalization based on the atomic capabilities concept has not been completely carried out.

For instance, [1] introduces dynamic aspects into the design of physical agents. Some approaches are introduced into this concept that takes dynamics into account to evaluate the difficulty of agent actions.

References [2] and [3] show their approach applied to a ball passing experiment between two robots. The purpose of the examples is to show the usefulness of inter-agent negotiation with explicit representations of dynamics and also to improve the decision of when and how to carry out the task with respect to static knowledge.

References [1], [4], [5], [6] show an example of convoying two autonomous mobile robots controlled as agents. The rear agent has the responsibility of avoiding collisions, but both are responsible for the reliability of secure decisions based on dynamics. The co-operative decisions based on dynamics provide the controllers with safer set points and a better coordinated control.

The aim of [7] is to find some attributes that describe the dynamics of the physical body of any agent, and to use them in a decision algorithm to let the agent know about its physical limitations.

According to [8], a good way to improve the cooperation performance between physical agents is to provide them with introspection about their physical bodies and their own limitations. This introspection allows the agents to manage their commitments better and avoid undesirable situations.

Reference [9] shows an example of a set of atomic capabilities and how it is a good way to represent knowledge related to the agent's physical body in order to improve the decisions making structure in a cooperative system. So, [9] addresses introspective reasoning in relation to these atomic capabilities to show how the performance of the multi-agent system is improved when the agents can manage their bodies by taking into account the atomic capabilities associated with their controllers.

An example of atomic capabilities is used in [10] and [11] in order to represent the dynamics of the physical agent as well as to generate and obtain dynamics diversity.

The capabilities seem to be the best way of representing the knowledge about the physical features of the systems in the above works, but it is still difficult to choose the information to include in these capabilities. In spite of this, it is possible to assume that the atomic capabilities have to be directly related to the controllers of the objects that the agents manage. Thus, it is possible to extract the information from the controllers in order to obtain a good physical knowledge of the agent's body. Along this research line, this paper attempts to formalize 
the atomic capabilities concept in a particular formulation for linear control systems. Specifically, this paper shows the impact of the atomic capabilities concept on the decisions making structure of physical agents when executing coordinated tasks. In this implementation both the passing a ball and the offside manoeuvre between physical agents in the robotic soccer testbed are used as coordinated tasks. This approach is extremely effective at the level of automatic control when it is necessary to have a decisions making structure about commitments between physical agents that takes into account dynamic aspects of their physical bodies in order to make physically feasible decisions and to get secure and reachable commitments.

Section II of this paper shows studies on the atomic capabilities concept applied to linear control systems. Section III shows the atomic capabilities approach in mobile robotics. Section IV gives an explanation of the study cases. Section V shows our approach to include control-oriented knowledge in the decisions making structure of physical agents. Section VI shows experimental results within this physical multi-agent environment. Finally, some conclusions are drawn in Section VII. Our tests were done using the robot models of the SimuroSot simulator available in the web page: http://www.fira.net/soccer/simurosot/overview.html. The simulator facilitates extensive training and testing of our learning methods

\section{AN ATTEMPT TO Formalize THE ATOMIC CAPABILITIES CONCEPT}

Physical agents that perform tasks in a multi-agent environment have to fulfil real time and real world requirements, such as situated behaviour, goal-oriented behaviour, efficiency and coordination. According to [1], physical agents must be built based on the Dynamical Physical Agents Architecture $\left(D P A^{2}\right)$. The $D P A^{2}$ is a layered architecture formed by three specialised modules (control, supervisor and agent). The control module is the direct connection between the agent and the real world. All the information about the environment and the agent's physical features are included in the atomic capabilities of this module. Within the physical features, the dynamics of the agents' physical bodies can be modified by means of their controllers. At a control level, physical agents can interact in the real world through a set of different controllers $\left(C_{1}, C_{2} \ldots C_{N}\right)$. Each controller does possible a different execution of the proposed tasks. Thus, the atomic capabilities $A C_{i} \forall i=1 \ldots N$, associated with a specific controller $C_{i}$, consist in a set of attributes that represents the dynamic and static features of the system's response using this controller.

According to [12], in the study and design of a control system based on the system's response, specifications are usually given in terms of the transient and the steady-state performance, and controllers are designed so that the specifications are all met by the designed system. Control engineers should know the specifications that the system's response must achieve before designing the controller for a system [13]. These specifications describe the response of the controlled system. They can be used to complete the atomic capabilities according to the control theory foundations. However, this information must be modified in order to accomplish the following requirements:

1. Knowledge in the atomic capabilities must be general and useful to represent any controlled system.

2. Atomic capabilities must allow computational treatment in order to be understandable for the agents.

3. Atomic capabilities must be comparable in order to be exploited as a decision tool by the agents.

The relevance of the atomic capabilities representation is related to the possibility of representing different automatic controllers with different control algorithms and different control laws of an effective fashion in order to be able to compare and use them as a decision tool and hence manage the agent's physical body in a better way.

The following atomic capabilities have been selected as a first attempt on which to base our approach for MultiInput Multi-Output (MIMO) linear control systems. (Note: $m$ represents the outputs number; $n$ represents the inputs number in all definitions).

Precision $\left(\delta_{A}\right)$ : This shows the capability of the controlled system to follow the changes of set point.

The precision is related to the error of the controlled system when it is excited by a ramp input signal $r(j, k)$ with slope $\tau$, after $2 \tau$ seconds. Let $y_{r}(j, k)$ be the ramp response and let $\tau$ be the time constant of the open-loop system taking into account the $\mathrm{k}$-th input and the $\mathrm{j}$-th output of the linear control system. Precision can be defined by (1).

$$
\begin{gathered}
\delta_{A}(\%)=\frac{\sum_{j=1}^{m} b_{i}(j) *\left(\frac{1}{\left.n-\sum_{k=1}^{n}\left(1-a_{\delta}(j, k)\right)_{k=1}^{n} a_{\delta}(j, k) * \delta_{A}(j, k) \%\right)}\right.}{m-\sum_{j=1}^{m}\left(1-b_{,}(j)\right)} \\
\delta_{A}(j, k)(\%)=100 \%-\lim _{i \rightarrow 2 \tau} \frac{r(j, k)-y_{n}(j, k)}{r(j, k)} \times 100 \%
\end{gathered}
$$

Overshoot $\left(\mu_{A}\right)$ : This is related to the maximum value reached by the transient response of the system with reference to the steady-state value. Let $y_{s}(j, k)$ be the unitstep response taking into account the $\mathrm{k}$-th input and the $\mathrm{j}$-th output. Let $y_{\max }(j, k)$ denote the maximum value of $y_{s}(j, k)$; and $y_{s s}(j, k)$, the steady-state value of $y_{s}(j, k)$. The overshoot is defined as $\mu_{A}(j, k)=y_{\max }(j, k)-y_{s s}(j, k) . \quad \mu_{A}$ is often represented as a percentage of the final value of the step response. This approach considers, like desirable condition, having a low $\mu_{A}$ capability as it is shown in (2).

$$
\begin{gathered}
\mu_{A}(\%)=\frac{\sum_{j=1}^{m} b_{\mu}(j) *\left(\frac{1}{n-\sum_{k=1}^{n}\left(1-a_{\mu}(j, k)\right)^{k} \sum_{k=1}^{n} a_{\mu}(j, k) * \mu_{A}(j, k) \%(\%)}\right.}{m-\sum_{j=1}^{m}\left(1-b_{\mu}(j)\right)} \\
\mu_{A}(j, k)(\%)=100 \%-\frac{\mathrm{y}_{\max }(j, k)-\mathrm{y}_{\mathrm{ss}}(j, k)}{\mathrm{y}_{\mathrm{ss}}(j, k)} \times 100 \%
\end{gathered}
$$

Speediness $\left(\sigma_{A}\right)$ : This represents the speed capability of the controlled system to reach the set point. The speediness is defined in (3) as a percentage relation between the 
settling time of the closed-loop system $t_{s}(j, k)$ and the settling time of the open-loop system $t_{s-o l}(j, k)$ taking into account $k$-th input and the $j$-th output.

$$
\begin{gathered}
\sigma_{A}(\%)=\frac{\sum_{j=1}^{m} b_{\sigma}(j) *\left(\frac{1}{n-\sum_{k=1}^{n}\left(1-a_{\sigma}(j, k)\right)^{n=1}} \sum_{\sigma}^{n} a_{\sigma}(j, k) * \sigma_{A}(j, k)(\%)\right.}{m-\sum_{j=1}^{m}\left(1-b_{\sigma}(j)\right)} \\
\sigma_{A}(j, k)(\%)=\left(1-\frac{t_{s}(j, k)}{t_{s-o l}(j, k)}\right) \times 100 \%
\end{gathered}
$$

If $\sigma_{A}(\%)>0$ for all controllers, then the fastest controller is that of highest percentage. In opposite case, the closed loop systems are slower than the open loop system and the highest negative percentage between them correspond to the fastest. Controlled systems with $\sigma_{A}(\%)>$ 0 always are faster than those with $\sigma_{A}(\%)<0$.

Persistence $\left(\gamma_{A}\right)$ : This shows the capability of the controlled system to follow the set point when there are external disturbances affecting the value of the system output. Rejecting disturbances is sometimes a specification of controller design and evaluating it depends on control engineer criteria. However, (4) and (5) provide the persistence for the two most common disturbances, the step signal and the pulse signal in the SISO case.

For the step disturbances:

$$
\gamma_{A}(\%)=\left[\left(1-\frac{I A E}{\tau * A}\right) \times(\% \text { disturbance })\right] \%
$$

$I A E$ is the integral absolute error $I A E={ }_{b}^{T}|e(t)| d t, \tau$ is the open-loop time constant, $A$ is the amplitude of the step disturbance and \%disturbance is the percentage of times that this disturbance affects the system.

For the pulse disturbances:

$$
\gamma_{A}(\%)=\left[\left(1-\frac{I A E}{B}\right) \times\left(\% \%_{-} \text {disturbance }\right)\right] \%
$$

$B$ is the pulse area, $\mathrm{B}=$ (amplitude)*(duration). If $\gamma_{A}(\%)<0$ then $\gamma_{A}(\%)=0 \%$, therefore, the system does not reject disturbances. If there is more than one kind of disturbance then the value of $\gamma_{A}(\%)$ will be the maximum value of the persistence of all of them. In (6) is shown the MIMO case.

$$
\gamma_{A}(\%)=\frac{\sum_{j=1}^{m} b_{y}(j) *\left(\frac{1}{n-\sum_{k=1}^{n}\left(1-a_{y}(j, k)\right)^{2} \sum_{k=1}^{n} a_{y}(j, k) * \gamma_{A}(j, k)(\%)}\right)}{m-\sum_{j=1}^{m}\left(1-b_{y}(j)\right)}
$$

Robustness $\left(\rho_{A}\right)$ : This describes the capability to maintain the output of the controlled system inside an acceptable range when there are internal disturbances in the system. The phase margin and gain margin provide a measurement of the system's stability. By taking into account these margins without variations in the open-loop system parameters $\left(M P(j, k)_{n o m}\right.$ and $\left.M G(j, k)_{n o m}\right)$ and with the maximum variations of these parameters $(M P(j, k)$ and $M G(j, k)$ ), taking into account the $\mathrm{k}$-th input and the $\mathrm{j}$-th output of the linear control system, the following robustness definition is proposed in (7).

$$
\begin{gathered}
\rho_{A}=\frac{\sum_{j=1}^{m} b_{\rho}(j) *\left(\frac{1}{n-\sum_{k=1}^{n}\left(1-a_{\rho}(j, k)\right)_{k=1}^{n} a_{\rho}(j, k) * \rho_{A}(j, k)}\right)}{m-\sum_{j=1}^{m}\left(1-b_{\rho}(j)\right)} \\
\rho_{A}(j, k)=\frac{\frac{M F(j, k)}{M F(j, k)_{n o m}}+\frac{M G(j, k)}{M G(j, k)_{\text {nom }}}}{2}
\end{gathered}
$$

Aggressiveness $\left(\alpha_{A}\right)$ : This shows how quickly the system responds in order to reach the set point. It is defined in (8) as the percentage relation between the rise time $t_{r}(j, k)$ and the settling time $t_{s}(j, k)$ using the k-th input and $\mathrm{j}$-th output of the linear control system.

$$
\begin{gathered}
\alpha_{A}(\%)=\frac{\sum_{j=1}^{m} b_{\alpha}(j) *\left(\frac{1}{n-\sum_{k=1}^{n}\left(1-a_{\alpha}(j, k)\right)^{k=1}} \sum_{\alpha}^{n} a_{\alpha}(j, k) * \alpha_{A}(j, k)(\%)\right)}{m-\sum_{j=1}^{m}\left(1-b_{\alpha}(j)\right)} \\
\alpha_{A}(j, k)(\%)=100 \%-\frac{t_{r}(j, k)}{t_{s}(j, k)} \times 100 \%
\end{gathered}
$$

Control Effort $\left(\varepsilon_{A}\right)$ : This describes the controlled system's effort to drive the output towards the desired value. The control effort is defined in (9).

$$
\begin{gathered}
\varepsilon_{A}=\sum_{j=1}^{m} b_{\varepsilon}(j) *\left(\frac{1}{\left.c s-\sum_{k=1}^{c s}\left(1-a_{\varepsilon}(k)\right)_{k=1}^{c s} a_{\varepsilon}(k) * \varepsilon_{A}(k)\right)}\right. \\
\varepsilon_{A}(k)=\frac{\downarrow_{1}^{k}\left|\frac{d u(k)}{d t}\right| d t}{u(k)_{\max }-u(k)_{\min }}
\end{gathered}
$$

$u(k)$ is the k-th control signal, $u(k)_{\max }$ denotes the maximum value of $u(k), u(k)_{\min }$ denotes the minimum value of $u(k), c s$ represents the control signals number. It is possible to obtain a comparative percentage between different controllers $C_{i}, \forall i=1 \ldots N$, about the $\varepsilon_{A}$ capability, following the considerations established by (10). In this sense, it has been assigned the highest percentage to the controller with the lowest $\varepsilon_{A}$ capability.

$$
\varepsilon_{A}(\%)=\frac{\min \left(\varepsilon_{A-C i}, \forall i=1 \ldots N\right)}{\varepsilon_{A-C i}} \times 100 \%, \forall i=1 \ldots N
$$

Coherence $\left(\chi_{A}\right)$ : This determines if the controllers have been designed to work according to the specifications that are requested, (e.g. point operation, work interval).

Control Kind $\left(\kappa_{A}\right)$ : This identifies the type of control that is being analysed. Thus, only controllers of the same kind are compared, (e.g. position controllers $\rightarrow \kappa_{A}=1$, velocity controllers $\rightarrow \kappa_{A}=2$, etc.).

The weight coefficients $\left(a_{\delta}, b_{\delta}, a_{\mu}, b_{\mu}, a_{\sigma}, a_{\mu}, b_{\gamma}, a_{\rho}\right.$ $\left.b_{\rho}, a_{\alpha}, b_{\alpha}, a_{\diamond}, b_{\varepsilon}\right)$ incorporated in the definitions mentioned 
before are useful to fix the relevance degree between $[0,1]$ of each k-th input and each j-th output when calculating the corresponding atomic capabilities, and therefore determining their relevance in the decisions making structure of the physical agent. Hence, the set of atomic capabilities $A C_{i}$ associated with the controller $C_{i}$ of the physical agent $A$ is: $A C_{i}=\left\langle\delta_{A}, \mu_{A}, \sigma_{A}, \gamma_{A}, \rho_{A}, \alpha_{A}, \varepsilon_{A}, \chi_{A}\right.$, $\left.\kappa_{A}\right\rangle, \forall i=1 \ldots N$. Below is an explanation of how we can use them in a particular implementation.

\section{ATOMIC CAPABILITIES FOR LINEAR CONTROL Systems APPliEd To MOBILE RoBotics}

We have used non-holonomic mobile robots to demonstrate our approach using a linearised second-order model of the mobile robot dynamics [14]. Thus, the robot movement $(x(t), y(t), \theta(t))$ is controlled such that the robot follows the horizontal axis $x$ with a constant linear velocity $v$. A control law based on the poles location method in which the values of the angular velocity $\omega$ are obtained in terms of the robot position $(y(t), \theta(t))$ is proposed in (11).

$$
\omega(t)=-\frac{\alpha_{1} \alpha_{2}}{v} y(t)+\left(\alpha_{1}+\alpha_{2}\right) \theta(t) ; \quad \alpha_{1,2}=-\zeta \omega_{n} \pm j \omega_{n} \sqrt{1-\zeta^{2}}
$$

$\alpha_{1,2}$ are the poles system, $\zeta$ is the damping factor and $\omega_{n}$ is the natural frequency of the characteristic equation of a second-order system. Thus, the linear controlled system for the movement variables $(y, \theta)$ of the robot can be written by using the Laplace's expressions (12) and (13).

$$
\begin{array}{r}
Y(s)=\frac{s^{2}+2 \zeta \omega_{n} s}{s^{2}+2 \zeta \omega_{n} s+\omega_{n}^{2}} y(0)+\frac{v s}{s^{2}+2 \zeta \omega_{n} s+\omega_{n}^{2}} \theta(0) \\
\theta(s)=\frac{s^{2}}{s^{2}+2 \zeta \omega_{n} s+\omega_{n}^{2}} \theta(0)-\left(\frac{\omega_{n}^{2}}{v}\right) \frac{s}{s^{2}+2 \zeta \omega_{n} s+\omega_{n}^{2}} y(0)
\end{array}
$$

Different dynamics can be designed using the above linearised model depending on the control engineer criteria. Figure 1 shows the step responses of this model for three different movement controllers $\left\{\mathrm{C}_{1}, \mathrm{C}_{2}, \mathrm{C}_{3}\right\}$.

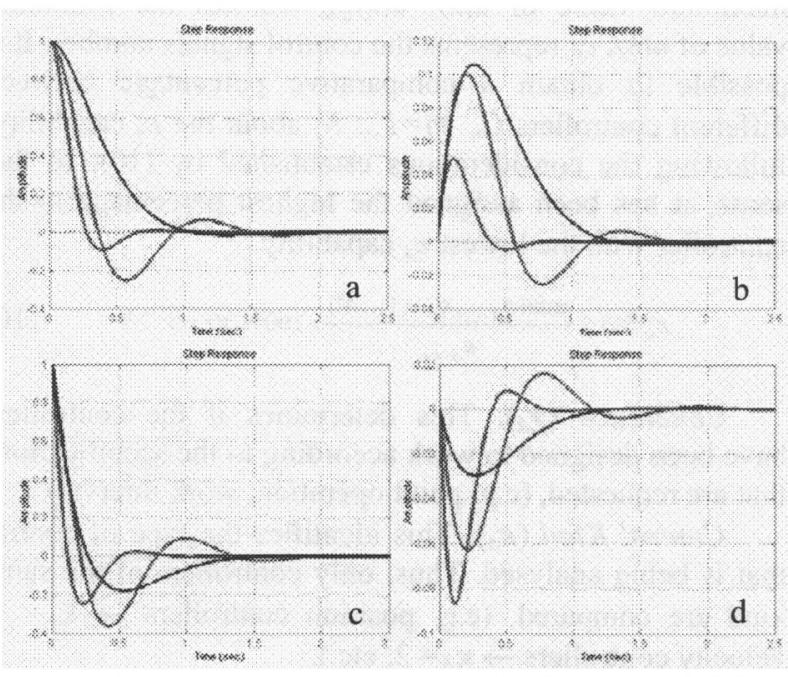

Figure 1: Step responses components: a). $\mathrm{Y}(\mathrm{s}) / \mathrm{y}(0) ; \mathrm{b}) . \mathrm{Y}(\mathrm{s}) / \theta(0)$; c). $\theta(\mathrm{s}) / \theta(0) ; \mathrm{d}) \cdot \theta(\mathrm{s}) / \mathrm{y}(0)$
It is possible to extract some atomic capabilities according to the definitions described in section II and the step responses shown in figure 1, and use them as a decision tool to select, for instance, the best controller to perform the proposed coordinated tasks. In this particular implementation all the weight coefficients are fixed to 1 to give the maximum relevance degree for each atomic capability component. Moreover, we have supposed that $100 \%$ of disturbances that affect the system are steps with amplitude $3.75 \mathrm{~cm}$ and duration 1 second (e.g. a probable crash with other mobile robot). Table I shows the atomic capabilities associated to each movement controller.

TABLE I

ATOMIC CAPABILITIES OF THE MOVEMENT CONTROLLERS

\begin{tabular}{|c|c|c|c|c|c|c|}
\hline Control & $\mu_{\mathrm{A}}(\%)$ & $\sigma_{\mathrm{A}}(\%)$ & $\gamma_{\mathrm{A}}(\%)$ & $\boldsymbol{\alpha}_{\mathrm{A}}(\%)$ & $\varepsilon_{\mathrm{A}}$ & $\boldsymbol{\kappa}_{\mathrm{A}}$ \\
\hline $\mathrm{C}_{2}$ & 77.83 & -42.57 & 57.54 & 93.14 & 81.62 & 1 \\
\hline $\mathrm{C}_{2}$ & 86.25 & 39.10 & 59.46 & 88.93 & 75.20 & 1 \\
\hline $\mathrm{C}_{3}$ & $\mathbf{8 7 . 4 8}$ & -4.03 & 57.08 & $\mathbf{8 0 . 3 0}$ & 100 & 1 \\
\hline
\end{tabular}

IV. OUR Proposed Study CASES

Different situations can appear in order to execute the same coordinated task in a multi-agent environment. These situations have to be taken into account when the physical agents make cooperative decisions. We have used some situations that are presented in the coordinated tasks explained below. Space limitations $S L$ (reduced space for movement due to the presence of other agents), motion disturbances $M D$ (collisions with other physical agents), time constraints $T C$ (deadlines in the tasks due to the environment dynamics), energy performance $E P$ (different energy expenses according to the tasks) and special behaviour (like aggressiveness $A B$ and quickness $Q B$ in the execution of the tasks) are considered as well as examining their combinations. Every combination of the situations has a priority-order $P O$ to establish the influence degree $I D$ of each one on the decisions of the agents. The sum of all IDs of the examined situations is equal to $1(100 \%)$.

In the first task two physical agents are involved. The passer must strike the ball towards the interception point in a suitable way. The shooter must intercept and shoot the ball with the intention of scoring in the opposite goal. Figure 2a shows an example of this task. It is possible to describe the overall state using the distance $D_{1}$ between the ball and the possible interception point $I P$, the initial speed of the ball Vo, which determines its behaviour, and the distance $D_{2}$ between the shooter and the IP. A more detailed explanation about this task can be found in [9]. According to the task requirements the variables involved with the physical and time constraints of the shooter are: the time needed to perform the task, the distance $D_{2}$ between the shooter and the $I P$, and the orientation of the shooter $\theta_{A}$. In order to use a more generic value, the orientation of the shooter is described in (14). Figure $2 b$ shows the variables that describe the shooter state.

$$
\theta_{\mathrm{A}}=\left|\alpha_{1}\right|+\left|\alpha_{2}\right|=\left|\theta_{\mathrm{L}}-\theta_{\mathrm{I}}\right|+\left|\theta_{\mathrm{L}}-\theta_{\mathrm{F}}\right|
$$

In the second task two physical agents are involved. Defender 1 and Defender 2 must coordinate between them 
to perform an offside manoeuvre and to avoid of this way the success of the passing a ball between two opposite physical agents. Figure 3 shows a situation example of this task. It is possible to describe the overall state using the time of the passer to strike the ball Time ${ }_{P}$, the distances $D_{3}$ and $D_{4}$ between each defender and the offside line as well as their respective generic orientations $\theta_{B}$ and $\theta_{C}$, (similar to $\theta_{A}$, but using as reference $O L$ ).

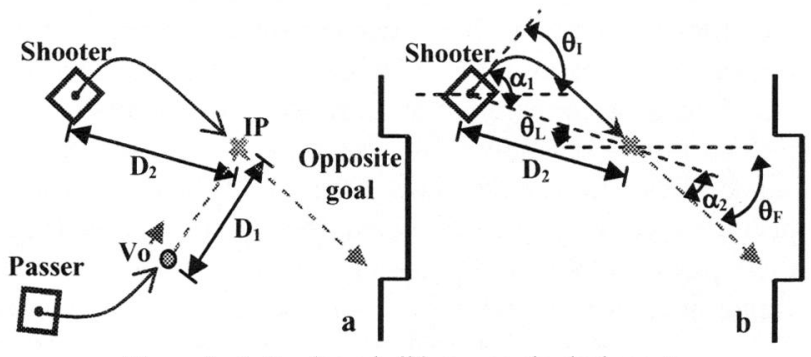

Figure 2. a). Passing a ball between physical agents

b). Variables that describe the shooter state

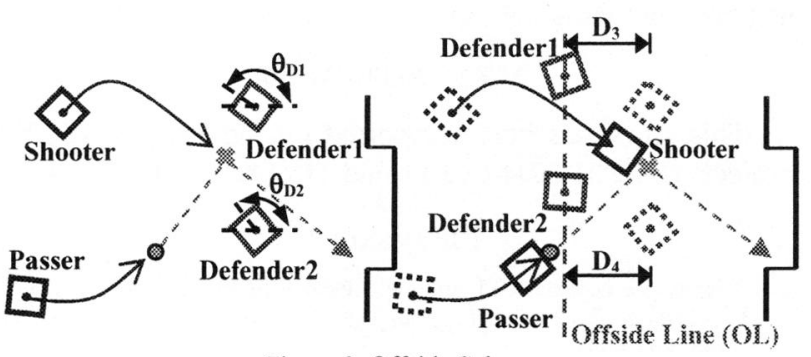

Figure 3. Offside Scheme

\section{ATOMIC CAPABILITIES APPROACH TO INCLUDE} CONTROL-ORIENTED KNOWLEDGE OF LINEAR CONTROL

\section{SYSTEMS IN THE DECISIONS OF THE PHYSICAL AGENTS}

Our proposal for including control-oriented knowledge in the decisions making structure of physical agents based on the atomic capabilities concept is drawn in figure 4 . This approach allows to the physical agent to be aware if it is able to do the expected task (introspective reasoning) by selecting the most suitable controller to perform it (managing the atomic capabilities associated with each controller)

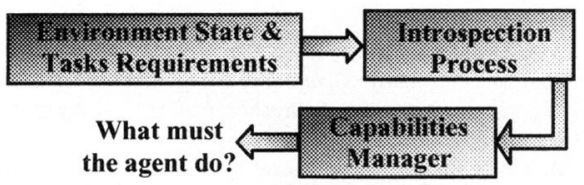

Figure 4. Scheme to obtain intelligent behaviours between agents.

For the first task the environment state is related to the shooter state $\left\{\mathrm{D}_{2}, \theta_{\mathrm{A}}\right\}$, the ball state $\left\{\mathrm{V}_{0}, \mathrm{D}_{1}\right\}$ and a combination of the situations described in section IV that we have called Case. The task requirements are related to the approach to task control and task execution described in [8] where a neural network $N N_{0}$ gives the execution time of the task Time $_{T}$. For the second task the environment state is related to the defenders state $\left\{\mathrm{D}_{3}, \theta_{\mathrm{B}}, \mathrm{D}_{4}, \theta_{\mathrm{C}}\right\}$, and the analysed Case. The task requirements are related to the needed time to perform the offside manoeuvre Time $_{P}$ and the offside line $O L$. The introspection process is implemented using neural networks $-N N_{l^{-}}$. This takes into account the environment state and the task requirements in order to give the agent's capability to perform the proposed task based on an execution certainty index $\left\{\mathrm{CI}_{\mathrm{Ci}}\right\}$ for each available controller. The resulting neural networks are not the result of an exhaustive search for the optimal configuration to suit these tasks, but rather the quickest and most successful of some alternatives with different numbers of hidden units and different learning rates. Figure 5 shows a scheme of our introspection process. Thus, the physical agent performs introspective reasoning in relation to its body to make individual and cooperative decisions in the tasks execution.

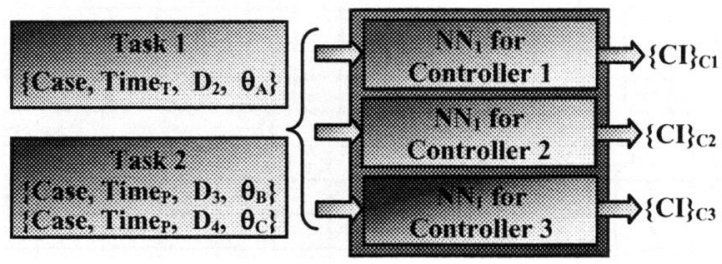

Figure 5. General scheme of our introspection process.

The capabilities manager decides which controller is suitable to execute the task according to the evaluation of their respective capabilities. Figure 6 shows a scheme of our capabilities manager. The Suitability Rates $\{\mathrm{SR}\}_{\mathrm{Ci}}$ of each controller for the proposed task are obtained according to their capabilities $A C_{i}$, the possibility of performing or not performing the task given by the execution certainty index $\{\mathrm{CI}\}_{\mathrm{Ci}}$ and the priority-order coefficients $P O$ of the involved situations in the analysed Case. Each atomic capability used in this approach has a direct relation with one studied situation, (e.g. $\mu_{A}$ with $S L$, $\gamma_{A}$ with $M D, \varepsilon_{A}$ with $E P$, etc.). The suitability rates are used in a decision maker in order to decide, if it is possible, the best controller to execute the task.

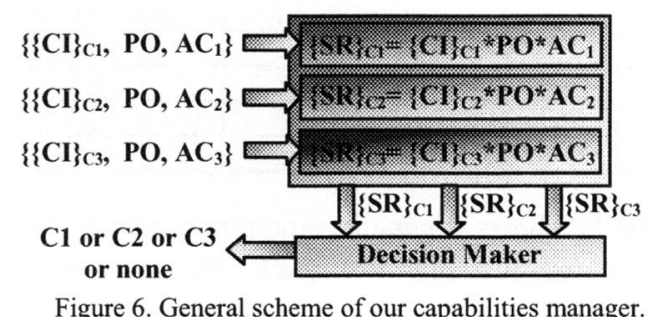

A high Suitability Rate indicates that the tasks can be performed. A low Suitability Rate indicates that the tasks cannot be performed. We have selected suitable decision thresholds in order to put this decisions making structure into practice. The task performance improves with this new and effective approach since the physical agents can make physically feasible decisions and get secure and reachable commitments according to control-oriented knowledge, as well as manage their physical bodies according to their capabilities. Thus, the physical agents have introspection about what they can and cannot do and how they are able to perform the tasks according to the knowledge about their physical features. 
VI

\section{EXPERIMENTAL RESULTS}

We have tested our approach using a typical scene that involve three aforementioned situations, $S L, M D$ and $E P$ using to this set a $P O=[40(S L) 0(Q B) 20(M D) 0(A B)$ $40(E P)] \%$ to be in agreement with the vector of atomic capabilities. Tables III shows the successful actions obtained when our physical agents perform the proposed coordinated tasks in different experiments (see Table II).

TABLE II

PROPOSED EXPERIMENTS FOR THE TASKS. (POS: POSITION VEL: VELOCITY, TRAJ: TRAJECTORY, F: FIXED, V: VARIABLE)

\begin{tabular}{|c|c|c|c|c|c|c|c|c|c|c|c|c|c|c|}
\hline \multirow{3}{*}{ 尊 } & \multicolumn{5}{|c|}{ Ball } & \multirow{2}{*}{\multicolumn{2}{|c|}{$\frac{\text { IP }}{P_{B S}}$}} & \multicolumn{3}{|c|}{ Passer } & \multicolumn{4}{|c|}{ Shooter } \\
\hline & Pos & \multicolumn{2}{|c|}{$\mathrm{Yel}$} & \multicolumn{2}{|c|}{ Traj } & & & \multicolumn{2}{|c|}{ Pos } & $\mathrm{Yal}$ & \multicolumn{2}{|c|}{ Pos } & \multicolumn{2}{|c|}{$\mathrm{Vel}$} \\
\hline & \begin{tabular}{l|l}
$f$ & $y$ \\
\end{tabular} & $f$ & $v$ & $f$ & $\mathrm{v}$ & $\mathrm{f}$ & $v$ & $\mathrm{f}$ & $\mathrm{v}$ & \begin{tabular}{l|l}
$f$ & $v$ \\
\end{tabular} & $\mathrm{f}$ & $y$ & f & $\mathrm{v}$ \\
\hline 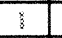 & $\cdot$ & & - & & $\cdot$ & $\cdot$ & & & - & - & & $\cdot$ & & • \\
\hline 2 & - & $\cdot$ & & & 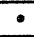 & & $\cdot$ & $\bullet$ & & - & & $\div$ & & $\because$ \\
\hline 3 & - & & - & & $\bullet$ & & $\bullet$ & & $\cdot$ & $\bullet$ & & $\div$ & & - \\
\hline \multirow{3}{*}{ 遌 } & \multicolumn{2}{|c|}{ OL } & \multicolumn{5}{|c|}{ Defender1 } & \multicolumn{4}{|c|}{ Defender2 } & \multicolumn{3}{|c|}{ Passer } \\
\hline & \multicolumn{2}{|c|}{ Pos } & & \multicolumn{2}{|c|}{ Pos } & \multicolumn{2}{|c|}{ Vel } & \multicolumn{2}{|c|}{ Pos } & \multicolumn{2}{|c|}{$\mathrm{Vel}$} & \multicolumn{3}{|c|}{ Time } \\
\hline & $f$ & $\mathrm{v}$ & & $f$ & $\mathbf{v}$ & $\mathrm{f}$ & $\mathbf{v}$ & $f$ & $\mathrm{v}$ & $\mathrm{f}$ & $\mathrm{v}$ & f & & $\mathrm{v}$ \\
\hline 3 & 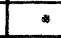 & & & & - & & $\cdot$ & & - & & . & & & - \\
\hline 2 & & $\bullet$ & & & - & & $\cdot$ & & $\cdot$ & & . & • & & \\
\hline 3 & & 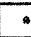 & & & - & & . & & $\cdot$ & &. & & & - \\
\hline
\end{tabular}

In the table III is possible to see how the performance is improved when our approach is used for individual and cooperative decisions in order to perform correctly the required tasks.

TABLE III

SUCCESSFUL ACTIONS IN THE PROPOSED TASKS

\begin{tabular}{|c|c|c|c|c|c|c|}
\hline \multirow{3}{*}{$\mid$} & \multirow{3}{*}{ 总产 } & \multicolumn{5}{|c|}{ Successful Scores } \\
\hline & & \multicolumn{3}{|c|}{$\begin{array}{c}\text { Individual } \\
\text { Performance }\end{array}$} & \multirow{2}{*}{$\begin{array}{l}\text { Random } \\
\text { Becision } \\
\{\mathrm{C}\}, \mathrm{C} 2, \mathrm{C} 3\}\end{array}$} & \multirow{2}{*}{$\begin{array}{c}\text { Oar } \\
\text { Approach } \\
\{\mathrm{C}, \mathrm{C} 2, \mathrm{C} 3\}\end{array}$} \\
\hline & & $\mathrm{Cl}$ & $\overline{\mathrm{C} 2}$ & $\mathrm{C} 3$ & & \\
\hline 1 & 180 & 345 & 382 & 437 & 330 & 449 \\
\hline 2 & 157 & 245 & 547 & 498 & 293 & 615 \\
\hline 3 & 231 & 845 & 896 & 1279 & 864 & 1323 \\
\hline \multirow{3}{*}{\multicolumn{2}{|c|}{$\begin{array}{l}\text { Case } \\
\text { Task } 2\end{array}$}} & \multirow{3}{*}{$\begin{array}{l}\text { Simu. } \\
\text { Number }\end{array}$} & \multicolumn{4}{|c|}{ Successful Offsides } \\
\hline & & & \multicolumn{2}{|c|}{$\begin{array}{l}\text { Colective Perfornance } \\
\text { Random Decision }\end{array}$} & \multicolumn{2}{|c|}{ 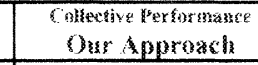 } \\
\hline & & & \multicolumn{2}{|c|}{$\mathrm{C}\{, \mathrm{C} 2, \mathrm{C} 3\}$} & \multicolumn{2}{|c|}{$\mathrm{C}\{, \mathrm{C} 2, \mathrm{C} 3\}$} \\
\hline$i$ & & 1152 & \multicolumn{2}{|c|}{148} & \multicolumn{2}{|c|}{456} \\
\hline 2 & & \multirow{2}{*}{$\frac{12969}{29489}$} & \multirow{2}{*}{\multicolumn{2}{|c|}{$\frac{250}{858}$}} & \multicolumn{2}{|r|}{522} \\
\hline 3 & & & & & \multicolumn{2}{|c|}{923} \\
\hline
\end{tabular}

Table IV shows the management rate of the controllers inside the agent's physical body for the task 1. The selected controller has the best performance for executing the proposed task. The physical agent really manages its behaviour controllers according to the information given by its atomic capabilities. Thus, a good management of the agent's physical body depending on its capabilities is in fact a good way to solve this decisions making problem and improve the performance of the multi-agent systems in coordinated tasks.

TABLE IV

MANAGEMENT RATES OBTAINED WHEN OUR PHYSICAL AGENT USES ITS CAPABILITIES MANAGER

\begin{tabular}{|c|c|c|c|c|}
\hline \multirow{2}{*}{ Case } & \multirow{2}{*}{$\begin{array}{c}\text { Successful } \\
\text { Scores }\end{array}$} & \multicolumn{3}{|c|}{ Controllers' Management Rate (\%) } \\
\cline { 3 - 5 } & $\mathrm{Cl}$ & $\mathrm{C} 2$ & $\mathrm{C3}$ \\
\hline 1 & 43 & 22.27 & 20.71 & 57.02 \\
\hline 2 & 315 & 12.78 & 43.02 & 44.20 \\
\hline 3 & 13.3 & 17.38 & 16.10 & 66.52 \\
\hline
\end{tabular}

\section{CONCLUSIONS}

This work shows that a good way of improving the cooperation performance between physical agents is to provide to them with introspection on the physical features of the physical objects (e.g. their physical bodies) that they handle, in order to manage their commitments better and to avoid possible undesirable situations. According to the above results, the atomic capabilities set is an effective tool for representing all knowledge related to the agent's physical features in order to improve the decisions making structure in a cooperative system. We have worked with introspective reasoning in relation to these atomic capabilities to show how the multi-agent system performance is improved when the agents can manage their physical objects by taking into account the atomic capabilities associated with their behaviour controllers. There are open studies on how to take advantage of this approach. Furthermore, to select one paradigm for the implementation of these concepts is not trivial at all, and its development is still open.

\section{ACKNOWLEDGMENT}

This work has been supported by the Spanish MCYT projects DPI2001-2094-C03-01 and TIN2004-06354-C02-02.

\section{REFERENCES}

[1] Oller A., de la Rosa J. LI. and Del Acebo E., DPA2: Architecture for Cooperative Dynamical Physical Agents. MAMAAW '99. Valencia Spain. June 1999

[2] de la Rosa J. Ll., García R., Innocenti B., Muñoz l., Figueras A., Ramón J. A., Rogi Team Real: Research on Physical Agents. 3rd Workshop on RoboCup, 16th IJCAI, July 31-August 6, 1999

[3] de la Rosa J. LI., Muñoz I., Innocenti B, Montaner M., Ramon J.A. Figueres A., RoGi Team Description, RoboCup-2000. LNAI 2019 Springer Verlag, pp. 551-554, Berlin (Germany). 2001.

4] Oller A., de la Rosa J. Ll., Innocenti B., Uso de Parámetros Dinámicos Para Mejorar la Cooperación entre Robots Móviles. Aplicación en un Convoy de Dos Robots. Seminario Anual de Automática, Electrónica Industrial e Instrumentación (SAAEl'99) pp. 311-314. Madrid - España.

5] de la Rosa J. L1. Innocenti B, Oller A., Figueres A., Ramon J.A., Muñoz I. Montaner M., An Example of Dynamical Physical Agents, 1st European Robocup Workshop, Amsterdam (Holland), 2000

[6] Ramón J. A., de la Rosa J. L1., Agentes Fisicos Dinámicos para Mejorar la Cooperación entre Robots Móviles. Tech Report. 2000.

[7] Innocenti B., de la Rosa J. Ll., Vehí J., Muñoz I., Montaner M., Fàbregas M., Figueras A., Ramon J. A., Examples of Dynamical Physical Agents for Multi Robot Control. 2do Workshop Hispano-Luso de Agentes Físicos Madrid 2001. Madrid-España.

[8] de la Rosa J., Quintero M. Christian G., Vehí J. About the Value of Introspection for Physically Grounded Commitments of Cooperative Intelligent Agents. In Proc. of V Workshop on Physical Agents, pp. 131 137. ISBN 84-933619-6-8, 2004

[9] Quintero M. Christian G., de la Rosa J., Vehi J. Physical Intelligent Agents' Capabilities Management for Sure Commitments in a Collaborative World. Frontiers in Artificial Intelligence and Applications. IOS Press, pp. 251-258. ISBN I 586034669.2004.

[10] Zubelzu J., de la Rosa J., Ramon J.A., Quintero M. Christian G. Managing Heterogeneity in a Robot Soccer Environment. Frontiers in Artificial Intelligence and Applications, IOS Press, pp. 317-322. ISBN I 586034669,2004

[11] Ramón J. A., de la Rosa J. Ll., Innocenti B., Estudio Preliminar sobre la Interacción de Agentes Fisicos Heterogénos, $3^{\circ}$ Workshop sobre Agentes Físicos Murcia 2002. Murcia - España. 2002

[12] Kuo B. C., Golnaraghi F., Automatic Control Systems. John Wiley \& Sons, Inc. $8^{\text {th }}$ Edition. ISBN 0-471-13476-7. 2003.

[13] Westphal L. C.. Handbook of Contro/ Systems Engineering. Kluwer Academic Publishers, $2^{\text {nd }}$ Edition. ISBN 0-7923-7494-0. 2001.

[14] Oller A.. García M. A., Using a Linear Model to Evaluate Performance of Non-holonomic Paths: The Shooting-Ball as a Case Studv. FIRA Robot World Congress 2002, pp. 643-647, 2002. 